\title{
Curcumin-artemisinin coamorphous solid and its preclinical and xenograft model
}

\author{
$\underline{\text { M K Chaitanya Mannava }}{ }^{1}$, Kuthuru Suresh ${ }^{1}$, Manish Kumar Bommaka ${ }^{1}$, Ashwini Nangia $^{1}$ \\ ${ }^{1}$ School Of Chemistry, University Of Hyderbad, Hyderbad, India \\ E-mail: mchaitanyasharma@gmail.com
}

Medicinal plants are gaining renowned interest in the scientific community for health benefits due to their reliable pharmacological actions. The search for anti-cancer agents from plant sources began in the 1950s. They have potential source of chemical constituents with antitumor and cytotoxic activities due to their natural antioxidants carrying out as reducing agents and free radical scavengers such as sequiterpene lactone, hydrophobic polyphenols that can reduce or minimize the toxic side effect of chemotherapy. Owing to this efficacy of natural extracts of plants now-a-day's open up for new prospect for cancer therapies. The most fascinating natural herbal extracts are curcumin, artemisinin, pyrogallol have diverse pharmacological effect such as antioxidant, antimalarial, antiproliferative, antiangiogenic, anticancer and gives a solution to the hurdles involved in chemotherapy.

Curcumin is a bright yellow colour phytochemical found in the rhizome of turmeric (Curcuma longa), chemically known as diferuloylmethane $(\mathrm{C} 21 \mathrm{H} 2006)$ is generally considered to be its most active constituent of curcuminiod and Artemisinin (approved by FDA as Antimalarial drug) is also a natural compound isolated from the plant Artemesia annua in 1972 by Youyou Tu (Nobel Prize 2015). Both the compounds are used as a traditional medicine in India and china for over 2000 years. Recently Padmanabhan et.al. reported synergistic effects by combining both the compounds Curcumin and Artemisnin and displayed enhanced antimalarial activity. In recent past curcumin and artemisinin possesses great attention for its potential therapeutic activity as an anticancer agent and revealed significant cytotoxic activities against a wide range of cancer cell lines including, melanoma, breast, pancreas, and renal cancer cells. Furthermore, xenograft experiments revealed its anticancer activity in pancreatic cancer.

However, the potential therapeutic efficacy of both CUR and ART is a thought-provoking today due to practical insoluble and shunned during tumour therapy because of drug interaction leads to poor bioavailability. A high level dose of drugs is always picked in invivo studies. As the high dosages doesn't meet patients compliances and the applications of such natural compounds in tumour therapy is hampered. Thus, in order to enhance the solubility and bioavailability, several formulations strategies are explored such as polymeric dispersion, nano crystals, supramolecular gelators, liposomes mixed, suspensions. From our group, recently reported various supramolecular solid forms i.e polymorphs, cocrystals and eutectics of curcumin. Among them Curcumin- Pyrogallol exhibited superior dissolution rate compared to all other cocrystals, eutectics and polymorphs. Later continued studies on curcumin with aim of enhanced bioavailability by making curcumin-artemisinin (CURART) coamorphous solid from fast evaporation under vacuum. It was showed good solubility and high pharmacokinetic profile in male sprague dwaley rats at $200 \mathrm{mg} / \mathrm{kg}$ than pure CUR (at $200 \mathrm{mg} / \mathrm{kg}$ dosage the plasma concentration not observed) and reported in RSC Advances (communication) journal. The present work evaluated the therapeutic activity that have associate with stability, bioavailability, toxicity and xenograft for superior dissolution rate of CUR-PYR and superior pharmacokinetic profile of CUR-ART coamorphous solids which is the subject matter of this poster.

(1) Suresh, K., Mannava, M.K.C., and Nangia, A (2014). RSC Advances, 4 (102), 58357-58361

(2) Chung et. al. (2010). Phytother. Res. 24, 189-192.

(3) Sanphui, P., Goud, N.R., Khandavilli, U.B.R., and Nangia, A. (2011). Cryst. Growth Des. 11, 4135-4145.

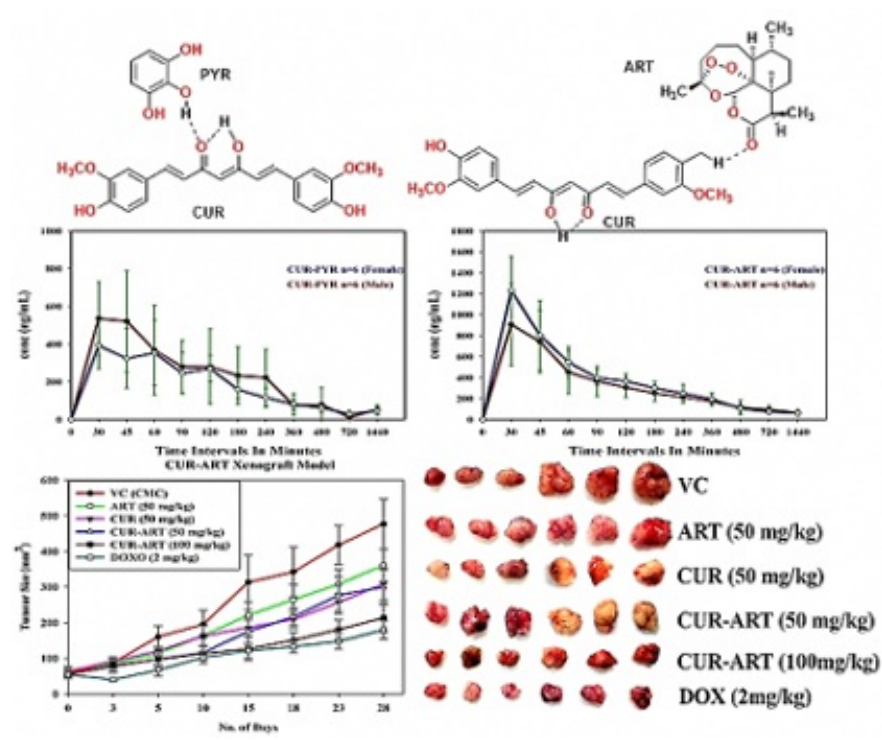

Keywords: Curcumin-Artemisinin, bioavailability, Xenograft-model 